\title{
An Experimental Study on Evaluating Glare in Blue Light Exposure
}

\section{$\operatorname{AUTHOR}(\mathrm{S}):$}

Miyazaki, Daisuke; Ueda, Kimi; Kawamoto, Soma; Takekawa, Wakako; Ishii, Hirotake; Shimoda, Hiroshi

\section{CITATION:}

Miyazaki, Daisuke ...[et al]. An Experimental Study on Evaluating Glare in Blue Light Exposure. Advances in Intelligent Systems and Computing 2020, 952: 103-112

\section{ISSUE DATE:}

2020

URL:

http://hdl.handle.net/2433/259832

\section{RIGHT:}

This is a post-peer-review, pre-copyedit version of an article published in Advances in Intelligent Systems and Computing. The final authenticated version is available online at: http://dx.doi.org/10.1007/978-3-030-20441-9_12.; This is not the published version. Please cite only the published version.; この論文は出版社版でありません。引用の際には 出版社版をじ確認じ利用ください。 


\title{
An Experimental Study on Evaluating Glare in Blue Light Exposure
}

\author{
Daisuke Miyazaki ${ }^{1}$, Kimi Ueda², Soma Kawamoto², Wakako Takekawa ${ }^{2}$, \\ Hirotake Ishii ${ }^{2}$, Hiroshi Shimoda ${ }^{2}$ \\ ${ }^{1}$ Faculty of Engineering, Kyoto University, Japan \\ ${ }^{2}$ Graduate School of Energy Science, Kyoto University, Japan \\ \{miyazaki, ueda, souma, tatekawa, hirotake,shimoda\}@ei.energy.kyoto-u.ac.jp \\ 606-8501, Yoshida Honmachi, Sakyo-ku, Kyoto, Japan
}

\begin{abstract}
It is known that blue light exposure to the eyes improves our arousal level. It is expected that exposure of office workers to blue light can maintain their concentration on their intellectual work and it may improve efficiency of their work. When blue light is exposed enough to improve arousal, however, it may cause feeling of dazzling and disturb their concentration on the contrary. In this study, therefore, an experiment was conducted to reveal the condition where they don't feel dazzling and their concentration is not disturbed, when changing the luminance and luminous area of blue light source. The participants performed cognitive tasks where blue light source was placed on their desks under one of four blue light conditions. As the result, it was found that the reasons why participants felt dazzling are asymmetrical intense light exposure and large luminous area.
\end{abstract}

Keywords: Blue light $\cdot$ Intellectual concentration $\cdot$ Melanopsin cells $\cdot$ Glare

\section{$1 \quad$ Introduction}

In recent years, they made efforts to improve intellectual productivity by improving the office environment. Many research studies on how to improve intellectual productivity in office work have been found [1][2]. For example, the studies focusing on the relationship between office room light and intellectual productivity have been made to propose lighting environments aiming at improving intellectual productivity [3]. On the other hand, some studies have revealed that blue light exposure improves human arousal [4]. The authors, therefore, have assumed that improvement of arousal by blue light exposure leads to an improvement of arousal and intellectual concentration, and an experiment was conducted to evaluate the intellectual concentration improvement effect by blue light exposure [5]. However, when exposing the blue light enough to improve arousal, the feeling of dazzling by the strong blue light sometimes disturb intellectual concentration. In order to reduce the glare and maintain the arousal enhancement effect by blue light exposure at the same time, it may be effective to increase the light emitting area and decrease the luminance in order to keep the total amount of blue light. In this study, therefore, an experiment was conducted to find the conditions that 
don't make them feel dazzling and disturb intellectual work with changing the luminance and the light emission area of blue light without changing the total amount of blue light to be exposed.

\section{Proposal of Blue Light Exposure to Improve Intellectual Concentration}

\subsection{Principle of intellectual concentration improvement by blue light exposure}

It had been supposed that there were two types of photoreceptors in the human retina which were rod cells and pyramidal cells. In recent years photoreceptors called melanopsin cells have been discovered as the third photoreceptor [6]. The spectral sensitivity of the melanopsin cells is shown in Fig. 1. As shown in Fig. 1, they have spectral sensitivity peaks around the wavelength of $480 \mathrm{~nm}$, that is, near the blue light wavelength. Therefore, melanopsin cells are thought to have a strong response to stimulation of blue light.

In addition, it has been proved that stimulated melanopsin cells improve human arousal [4]. The results of the study suggest that when blue light is projected on the retina, the melanopsin cells are stimulated and arousal is improved. Taking into consideration that arousal is a state in which the brain actively works, it is expected to improve concentration of intellectual work when the arousal is improved. Based on the above, the authors have assumed that exposure to blue light improves arousal, and intellectual concentration is improved as a result.

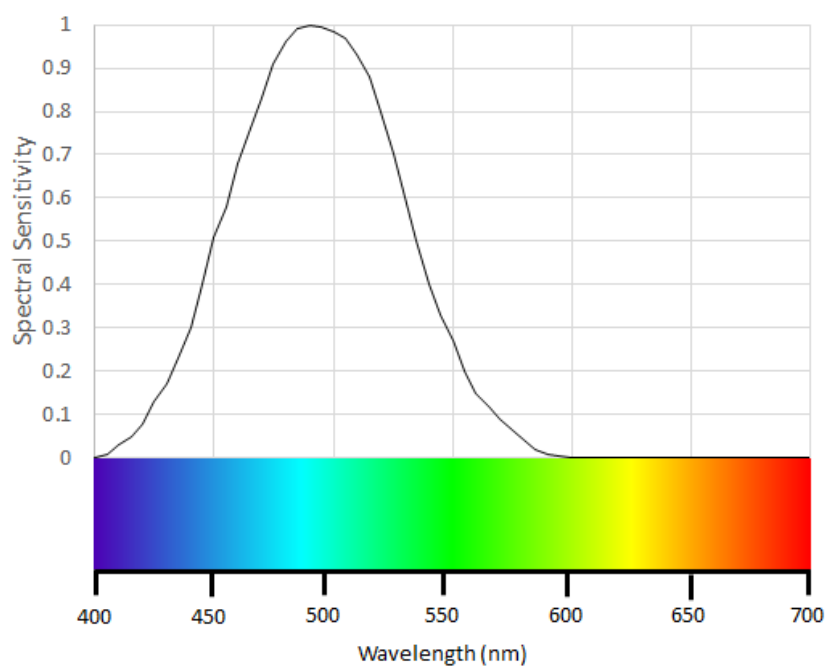

Fig. 1. Spectral Sensitivity of Melanopsin Cells and Wavelength of Color. 


\subsection{Design of blue light source}

A blue light source was designed which dimensions are a width of $95 \mathrm{~mm}$, a height of $200 \mathrm{~mm}$, and a depth of $100 \mathrm{~mm}$ for easy installation for practical desk work. The shape of light emitting surface was a rectangle with a length of $145 \mathrm{~mm}$ and a width of $80 \mathrm{~mm}$. Regarding the intensity of blue light, based on the spectral sensitivity of the melanopsin cell shown in Fig. 1, the intensity of the blue LED was determined so as to be equivalent to the stimulus that they receive as the same amount for white light with illuminance $3000 \mathrm{~lx}$ and color temperature $5000 \mathrm{~K}$. According to Weber-Fechner's law, human being perceives an external stimulus with a sensory quantity proportional to the logarithm of the intensity of the stimulus. Therefore, by setting the light conditions that increase the luminance exponentially, it is considered that the difference in brightness perceived under each light condition is constant. Base on the above consideration, the luminance of the blue light was designed so that the output can be changed in four stages of $100 \%, 50 \%, 25 \%$, and $12.5 \%$ with the luminance of the standard output. As the light conditions, 1 unit luminance $100 \%$ output, 2 units luminance $50 \%$ output, 4 units luminance $25 \%$ output and 8 units luminance $12.5 \%$ output were set in order to

keep total amount of blue light exposure. In the light condition of 1 unit luminance $100 \%$ output, the light source was placed diagonally on the left of users as shown in Fig. 2(a). Under other light conditions, they were placed symmetrically on the left and right front of users as shown in Fig. 2 (b)(c)(d).

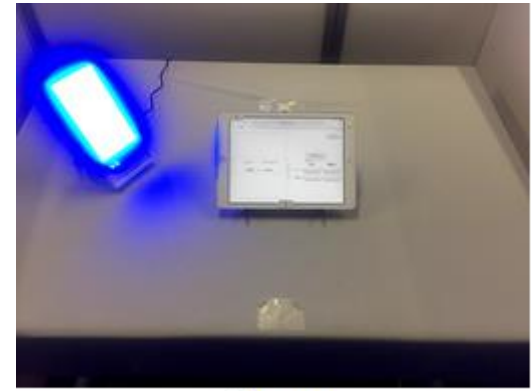

(a) 1 unit

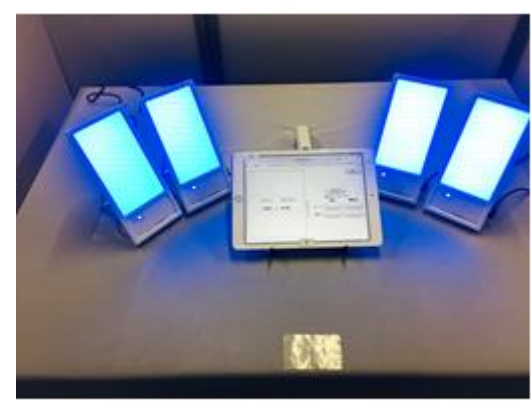

(c) 4 units

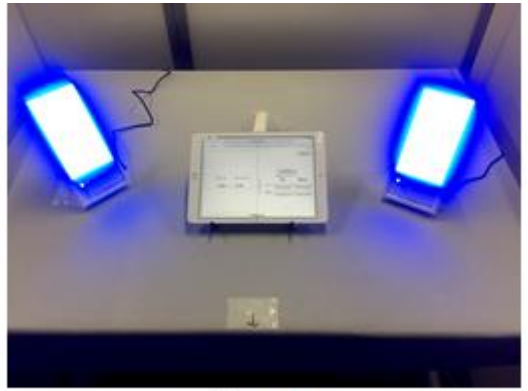

(b) 2 units

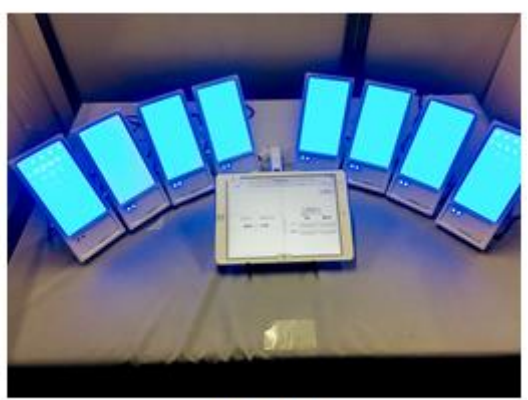

(d) 8 units

Fig. 2. Placement of blue light sources under each condition. 


\section{Glare Evaluation Experiment}

\subsection{Purpose and outline of experiment}

The purpose of this experiment is to investigate which blue light condition does not cause feeling of dazzling or disturb intellectual concentration. The experiment was conducted in the environmental control laboratory in Kyoto University from November 22 nd to 26th in 2018. As the experimental conditions, the four light conditions as shown in Fig. 2 were set and each participant performed a cognitive task called 'comparison task' under the light conditions in random order. At the end of the task under each condition, a glare questionnaire was conducted to subjectively evaluate the glare, and another questionnaire was conducted to subjectively evaluate their fatigue, arousal and concentration. At the end of the experiment, a final questionnaire was conducted to investigate which light condition was distractive while doing tasks and which light conditions was not acceptable for work.

\subsection{Methods}

As an intellectual task to measure the subjective evaluation of glare, a comparison task which requires language processing ability and numerical processing ability often employed in actual office work was assigned to the participants. The screen of the task is shown in Fig. 3. The comparison task is a cognitive task that simultaneously compares the meaning categories of the two words and compares two numbers displayed on iPad. In the word comparison, two words belonging to one of the four categories of (i)place name, (ii)artifact, (iii)animal and (iv)plant are displayed, and they have to judge whether those words belong to the same category or not. On the other hand, in numeric comparison, two of four-digit numbers are displayed with an inequality sign and they have to judge whether the inequality is correct or not. When they tap the button corresponding to the combination of these two comparison answers, the next question is displayed. The participants were asked to solve it one after another until the task time was over.

The environmental conditions of the experimental room are shown in Table 1. The temperature and humidity were kept constant by using air conditioning equipment and 4 circulators. The ventilation was also controlled in order to keep the carbon dioxide concentration under $1200 \mathrm{ppm}$. 


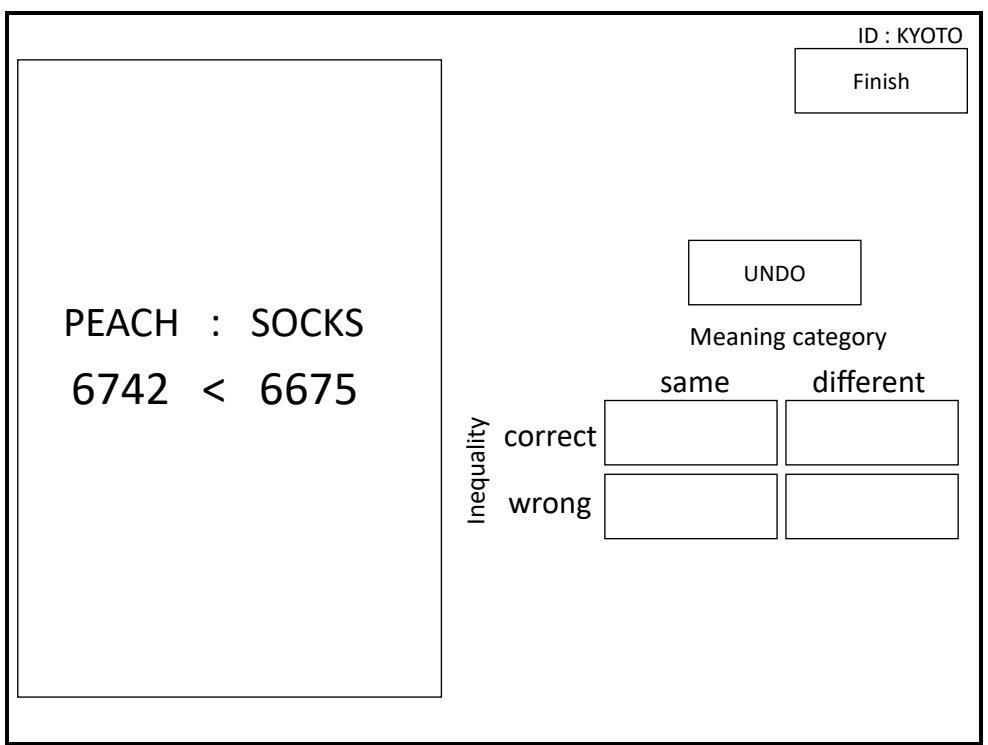

Fig. 3. Screen of Comparison Task.

Table 1. Environmental Conditions of Experimental Room

\begin{tabular}{ll}
\hline \multicolumn{1}{c}{ Environmental factor } & \multicolumn{1}{c}{ Value } \\
\hline Temperature & $23.5 \pm 0.5^{\circ} \mathrm{C}$ \\
Humidity & $35 \pm 5 \%$ \\
$\mathrm{CO}_{2}$ concentration & Under $1200 \mathrm{ppm}$ \\
Desk surface illuminance & $650 \pm 501 \mathrm{x}$ \\
\hline
\end{tabular}

The experiment schedule is shown in Fig. 4. 5-minute practice SET was prepared for the participants to become accustomed to the comparison task. While practicing it they experienced all the blue light conditions in the order from low luminance to high luminance for about 1 minute each. At the end of each task SET, they answered the regular questionnaire and the glare questionnaire. After completing all the task SETs, they experienced all the light conditions again and answered the final questionnaire. Since there were four types of blue light conditions, the order of the blue light conditions in each SET was exchanged depending on the participants in order to take the counterbalance of the order effect of the conditions. 


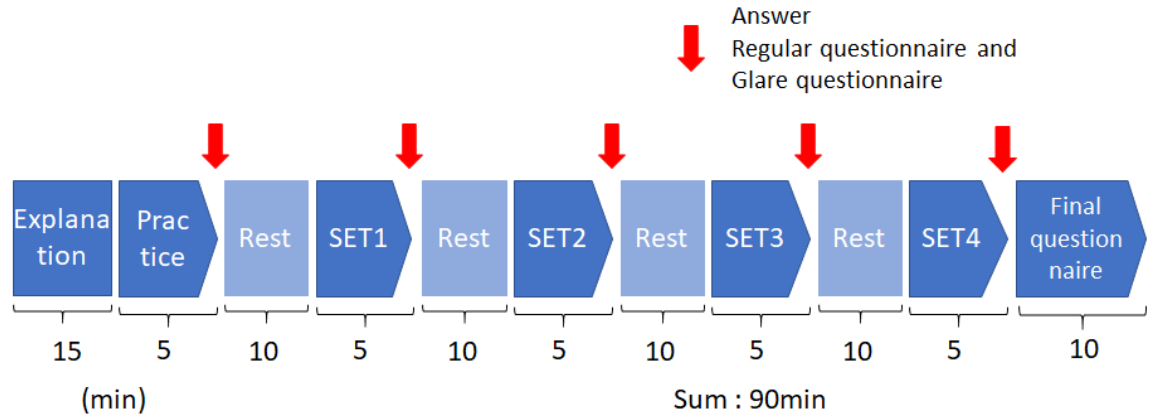

Fig. 4. Schedule of Experiment.

As an evaluation index, subjective dazzling was measured using the glare questionnaire. Subjective fatigue, subjective arousal, degree of concentration were also measured using the regular questionnaire to check whether there was any physical influence under each light condition. The glare questionnaire asked subjective dazzling of each blue light condition with 9 grades referring to BGI (British Daylight Glare Index). The grades were "1: Imperceptive, 3: Perceptible but not unacceptable, 5: Unacceptable but not uncomfortable, 7: Uncomfortable but not intolerable, 9: Intolerable". The regular questionnaire asked subjective fatigue, refreshment of brain and concentration with a numerical value from 0 to 100 after each task SET.

At the end of the experiment, they experienced all the blue light conditions again and then answered the final questionnaire. It asked them which light conditions were distractive while doing the task and were not acceptable with free description of their reasons.

The participants were 25 university students of Kyoto University. They are healthy males of the ages from 19 to 26 , with normal eye sight.

\subsection{Results}

Fig. 5 shows the results the average answers of the glare questionnaire depending on the task SETs and the blue light conditions. Regarding to the task SETs, the subjective dazzling of the task SET 1 was the most, while it was reduced from SET 1 to SET 3. In the last SET, it increased again. Regarding the blue light conditions, on the other hand, the feeling of dazzling under the condition of 1 unit luminance $100 \%$ output was the most, while the least was that under the condition of 2 units luminance $50 \%$ output. Those under other two conditions of 4 units luminance $25 \%$ output and 8 units luminance $12.5 \%$ output were almost the same.

Regarding the regular questionnaire, no significant difference was found in all the items between the task SETs and the blue light conditions

Table 2. shows the answers of the final questionnaire and the summary of the reasons for the response. Regarding "Which light conditions are distractive while doing the task", the number of participants who selected the light condition of 1 unit luminance $100 \%$ output was the most, and as the number of units increased, the number of answers decrease. According to the free descriptions following this question, there were many opinions that they felt dazzling under the light condition of 1 unit luminance $100 \%$ 
output because intense light was exposed asymmetrically only from their left side. As for "Which light conditions are unacceptable for work," the answer of the light condition with 1 unit luminance $100 \%$ output was the most, and the light condition with 8 luminance $12.5 \%$ output was the second. As for the reason why the light condition of 1 unit luminance $100 \%$ output is intolerable, many opinions were obtained as the same as the above. On the other hand, the reason why the light condition of 8 units luminance $12.5 \%$ output was not acceptable neither was mainly because the light emitting area was large and it made them feel dazzling. The number of participants who answered the light condition of 2 units luminance $50 \%$ output was intolerable was the least among all the light conditions.

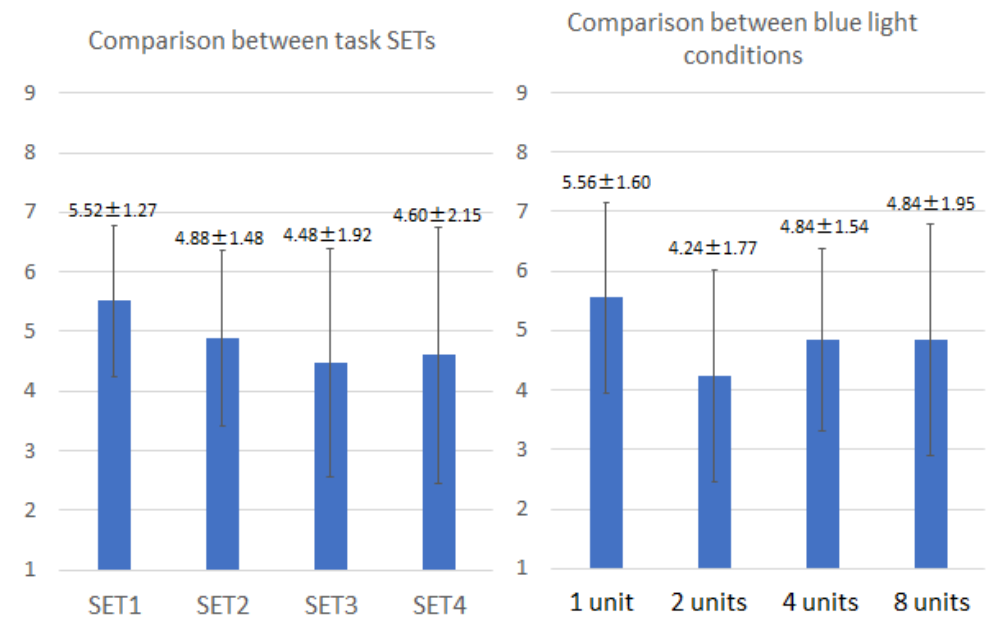

Fig. 5. Result of Glare Questionnaire. 
Table 2. Answer of Final Questionnaire

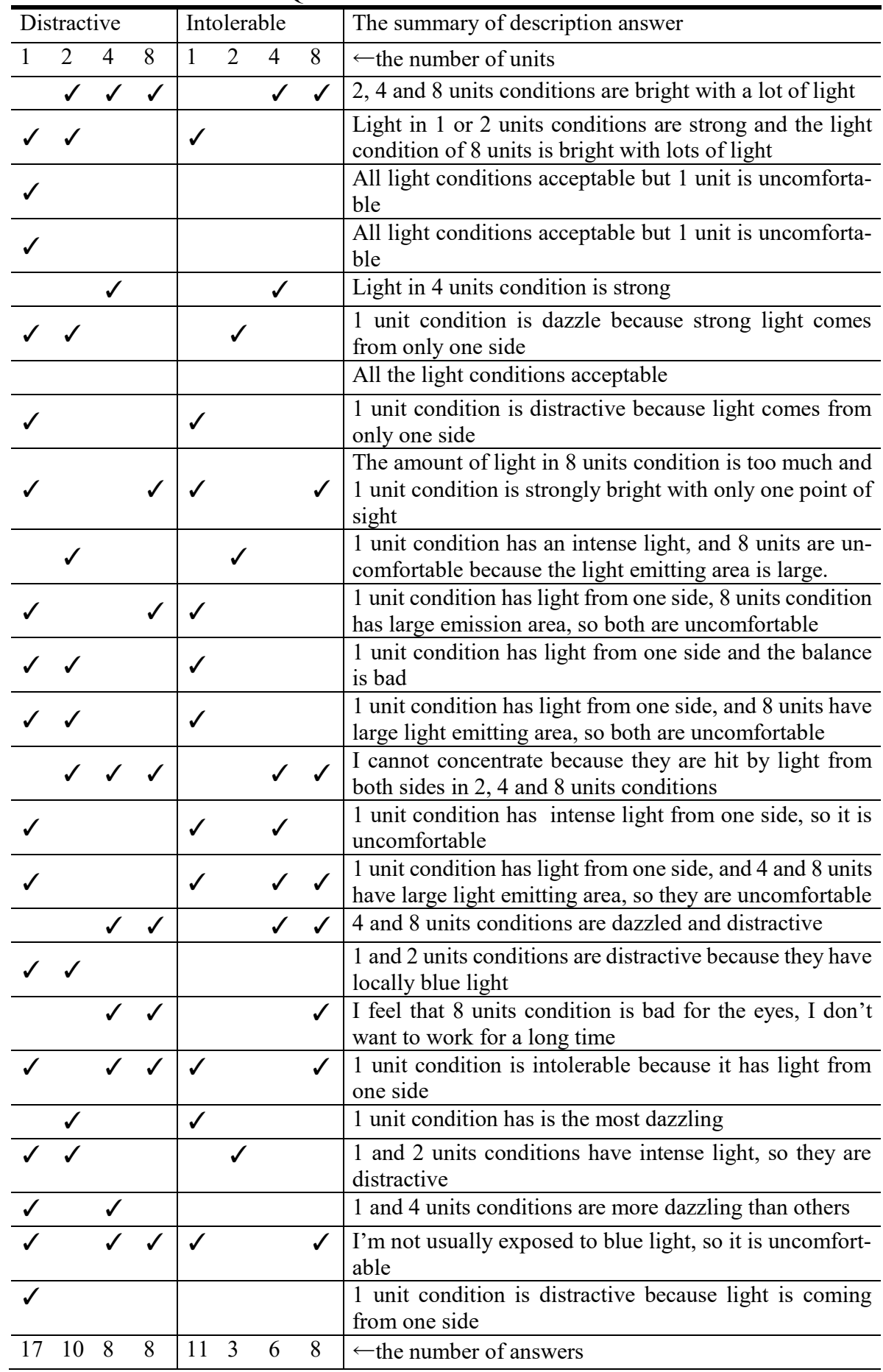




\subsection{Discussions}

Regarding the glare questionnaire, the answer of SET 1 obtained the most between the task SETs The participants experienced the blue light exposure only for the last 5 minutes of the practice SET, and it was supposed that they were not yet accustomed to the blue light and they felt excessively dazzling in SET 1. In comparison between the blue light conditions, a large difference was found between the light condition of 1 unit luminance $100 \%$ output and that of 2 units luminance $50 \%$ output.

Regarding the regular questionnaire, no significant difference was found in the influence on fatigue, refreshment of brain and concentration between the blue light conditions.

Regarding the final questionnaire, there were the most participants who answered that 1 unit luminance $100 \%$ output was intolerable and the least who answered that 2 units luminance $50 \%$ output. This result was consistent with the result of the glare questionnaire. The reasons why they answered "It is distractive while doing tasks" or "It is not acceptable for work" under the light condition of 1 unit luminance $100 \%$ output are that the blue light was exposed asymmetrically from only their left side and it was intense. In addition, regarding the light condition of 8 units luminance $12.5 \%$ output, many opinions were found that large light emitting area was uncomfortable.

Based on the above results of the glare questionnaire and the final questionnaire, in order not to disturb the intellectual work in the blue light exposure with keeping the effect of arousal enhancement, it is necessary to symmetrically irradiate blue light of low luminance from both sides of the field of view and to reduce the light emitting area. If the luminance or the light emission area is reduced to suppress the glare, the effect of arousal enhancement by the blue light exposure may be diminished. In order to realize a blue light exposure environment for intellectual concentration improvement, therefore, it is necessary to balance the luminance and light emission area to an appropriate value within a range obtaining the effect of arousal enhancement without causing dazzling.

\section{Conclusion}

In this study, the authors conducted an experiment to measure the subjective evaluation of glare to find what kind of blue light condition did not cause feeling of dazzling and disturb intellectual work. As the result, three factors of luminance, light emitting area, and asymmetric irradiation of light were found as the factors that caused feeling of dazzling. In other words, they feel dazzling when the luminance was high, the emission area was large, or when the light emitted asymmetrically. Therefore, it is supposed that the blue light exposure that does not disturb the intellectual work and improve arousal is that the luminance is low to some extent, the emission area is small, and the light is exposed symmetrically. In the future, with reference to this experimental result, it is necessary to conduct another experiment to confirm that an appropriate blue light exposure can improve intellectual concentration with the effect of arousal enhancement.

Acknowledgments. This work was supported by JSPS KAKENHI Grant Number JP17H01777. 


\section{References}

1. Haneda, M., Nishihara, N., Kawaguchi, G., Tanabe, S.: Productivity in Office with Elevated Preset Temperature in Summer -Improvement of thermal satisfaction by cooling methods and its effect on performance and fatigue-. In: J. Envision, Eng. AIJ, Vol. 74, No. 646, pp. 132137 (2009)

2. Virgnia, L., Caroline, P., Georgia, G.: Interior Plants May Improve Worker Productivity and Reduce Stress in a Windowless Environment. In: Journal of Environmental Horticulture, Vol. 14, No. 2, pp. 97-100 (1996)

3. Ishii, H., Kanagawa, H., Shimamura, Y., Uchiyama, K., Miyagi, K., Obayashi, F., Shimoda, H.: Intellectual productivity under task ambient lighting. In: Lighting Research and Technology, Vol. 50, pp. 237-252 (2018)

4. Yuda, E., Ogasawara, H., Yoshida, Y., Hayano, J.: Enhancement of autonomic and psychomotor arousal by exposures to blue wavelength light: importance of both absolute and relative contents of melanopic component. In: Journal of Physiological Anthropology, Vol. 36, No. 13 (2017)

5. Miyazaki, D., Ueda, K., Kawamoto, S., Takekawa, W., Ishii, H., Shimoda, H., Yabuki, J., Uchida, T., Noguchi, H.: An Experimental Study on Intelligent Concentration Improvement by Blue Light Exposure. In: Proc., Human Interface Symposium 2018 (2018) (In Japanese)

6. Provencio, I., Jiang, G., Grip, W., Hayes, W., Rollag, D.: Melanopsin: An opsin in melanophores, brain and eye. In: PNAS, Vol. 95, No. 1, pp. 340-345 (1998) 